\section{Signo de Leser-Trélat asociado a adenocarcinoma gástrico. Caso clínico}

\author{
FELIPE VENEGAS A. ${ }^{1}$, PAULA VACCARO M. ${ }^{1}$, \\ GABRIEL ABUDINÉN A. 2,a, CARLOS REYDET V. ${ }^{1}$, \\ FRANCISCO BRUNIE V. ${ }^{3}$, JAVIER ARCUCH D. ${ }^{4}$
}

\section{Leser-Trélat sign associated with gastric cancer. Report of one case}

We report a 66-year-old male presenting with malaise, heartburn and pruritic seborrheic keratoses in both feet of sudden onset, suggesting a Leser-Trélat sign. An upper gastrointestinal endoscopy disclosed a gastric cancer. The patient was subjected to a total gastrectomy and during follow up, the skin lesions had disappeared.

(Rev Med Chile 2012; 140: 1585-1588).

Key words: Foot dermatoses; Paraneoplastic syndroms; Stomach neoplasms.
'Servicio Urgencias, Hospital Víctor Río Ruiz, Los Ángeles, Chile. ${ }^{2}$ Facultad de Medicina y Biociencias, Universidad San Sebastián

Concepción, Chile. ${ }^{3}$ Servicio Medicina Interna,

Hospital de Mulchén, Los Ángeles, Chile. ${ }^{4}$ Servicio Cirugía, Hospital Víctor Río Ruiz,

Los Ángeles, Chile. IInterno VII Año Medicina.

Recibido el 1 de diciembre de 2011, aceptado el 9 de agosto de 2012

Correspondencia a: Drv: Gabriel Abudinén Adauy E-mail:gabriel_obl@ hotmail.com
$\mathrm{E}$ 1 cáncer gástrico es el segundo cáncer más común en el mundo con 934.000 casos nuevos por año en $2002^{1}$. Su incidencia varía en forma importante de un país a otro, y Chile se cuenta entre los países con las tasas más altas, junto a Japón, Costa Rica y Singapur. En nuestro país, representa la primera causa de muerte por tumores malignos para ambos sexos ${ }^{2}$.

Las metástasis a nivel hepático y pulmonar son aproximadamente el $40 \%$ de los casos, y el peritoneo y los huesos en alrededor de $10 \%{ }^{3,4}$. Pacientes con adenocarcinoma gástrico pueden manifestarse también con condiciones paraneoplásicas: síndrome de Trousseau (trombosis), acantosis nigricans, nefropatía membranosa, anemia hemolítica microangiopática, signo de Leser-Trélat (LT) (queratosis seborreica), dermatomiositis ${ }^{4,5}$.

Dentro de los signos paraneoplásicos más controversiales se encuentra el LT, el que es descrito como la aparición súbita e incremento en tamaño y número de múltiples queratosis seborreicas (QS) en asociación con una neoplasia interna ${ }^{5}$. La mayoría de los casos que han sido reportados son adenocarcinomas (especialmente estómago, colon y mamas), en menor número casos asociados a linfoma/leucemia, carcinoma de células escamosas y malignidades hematológicas ${ }^{6}$.

El 20\% de pacientes con el signo de Leser -
Trélat presenta acantosis nigricans concomitantemente, y $43 \%$ presenta prurito asociado, siendo la localización más frecuente la espalda ${ }^{5,6}$.

Comunicamos un caso en el cual se presentó el signo de Leser-Trélat como motivo de consulta en un paciente con adenocarcinoma gástrico.

\section{Caso clínico}

Paciente 66 años de edad, sexo masculino, con antecedentes mórbidos de hipertensión arterial y diabetes mellitus (DM) tipo 2 en tratamiento. Ingresa a servicio de cirugía del Hospital Base de Los Ángeles por cuadro clínico de 4 meses de evolución caracterizado por astenia, adinamia, ardor epigástrico, pirosis y regurgitación, asociado a lesiones dérmicas, de aparición súbita en ambos pies, no pruriginosas. El examen físico reveló un paciente en buen estado general, en el que destacaba únicamente presencia de lesiones queratócicas, seborreicas e hiperpigmentadas en ambos pies (Figuras 1 y 2) lo cual asociado a historia clínica sugería signo de Leser-Trélat.

Por sospecha de neoplasia del tubo digestivo se inició estudio, exámenes de laboratorio no evidenciaron alteraciones, se realizó endoscopia digestiva alta concluyendo presencia de lesión 


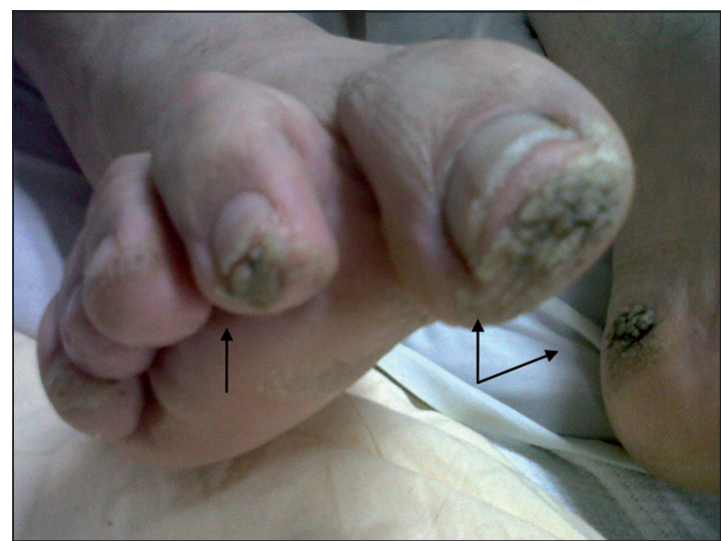

Figura 1. Lesiones de Leser-Trélat a nivel de extremos distales de ortejos pie derecho y talón izquierdo.

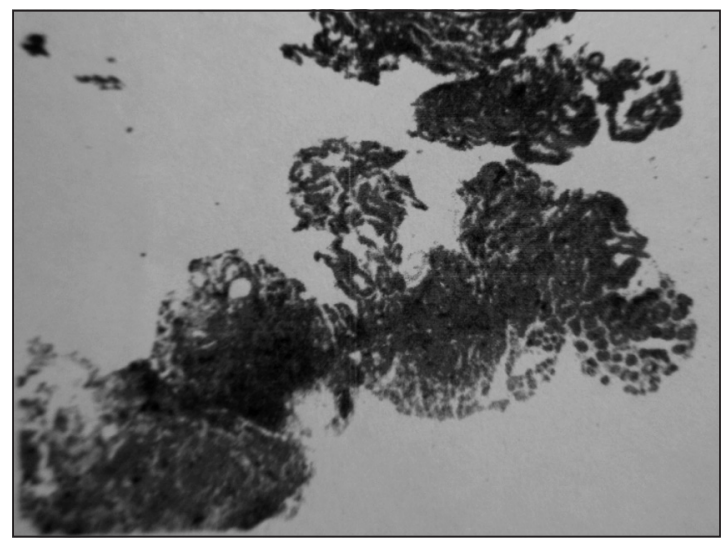

Figura 3. Se identifican fragmentos de mucosa gástrica con infiltración por neoplasia de tipo epitelial.

ulcerada cuerpo gástrico, la cual se biopsió y test de ureasa con resultado positivo para $H$. pylori. Posteriormente, informe de biopsia concluyó lesión de aspecto morfológico compatible con fragmento de mucosa gástrica infiltrada por adenocarcinoma moderadamente diferenciado con signos de ulceración activa (Figuras 3 y 4 ).

Por lo anterior, se inició estudio de diseminación y preoperatorio dentro de los cuales se solicitó tomografía axial computarizada (TAC) de abdomen y pelvis no evidenciando enfermedad macroscópica a distancia ni adenopatías, angiotomografía computada (Angiotac) multicorte tóraco-abdominal sin compromiso tumoral a grandes vasos, radiografía de tórax antero-posterior y lateral sin

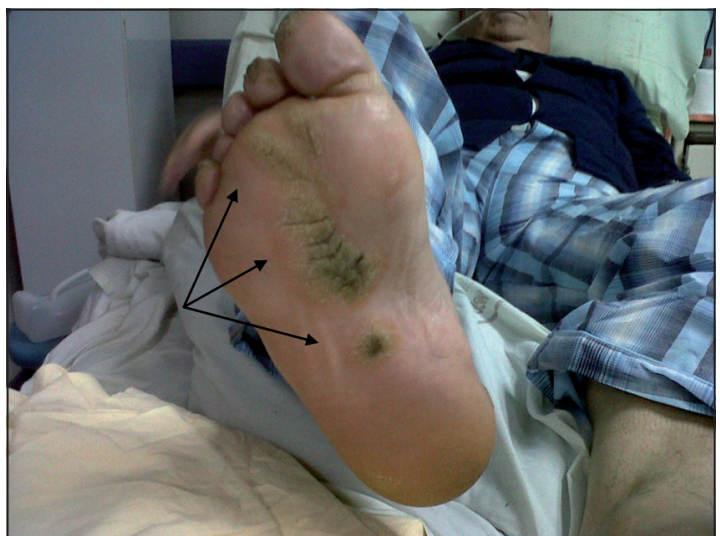

Figura 2. Lesiones de Leser-Trélat a nivel de cara plantar pie derecho.

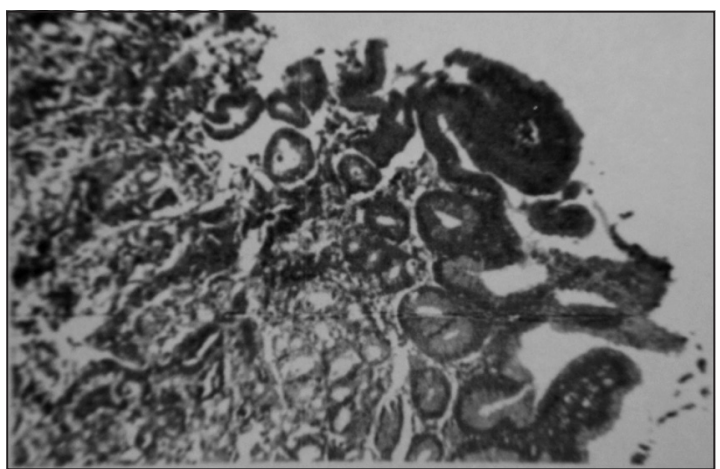

Figura 4. Se observa neoplasia de tipo epitelial, moderadamente diferenciada, con presencia de brotes celulares disgregados y formación tubulares irregulares, pequeñas.

alteraciones significativas, electrocardiograma y ecocardiograma sin contraindicación quirúrgica.

Debido a que estudio de diseminación no evidenciaba compromiso metastásico y contaba con criterios de operabilidad se decidió tratamiento con intención curativa, realizándose gastrectomía total ampliada (Figura 5) más colecistectomía por equipo de Cirugía Digestiva Alta del Hospital Base de Los Ángeles, Víctor Río Ruiz. Posteriormente, el paciente fue dado de alta, acudiendo a controles seriados en policlínico del hospital. El último control fue poco antes de escribir esta comunicación, presentando una evolución favorable con desaparición de las manifestaciones cutáneas (Figuras 6, 7a y 7b). 


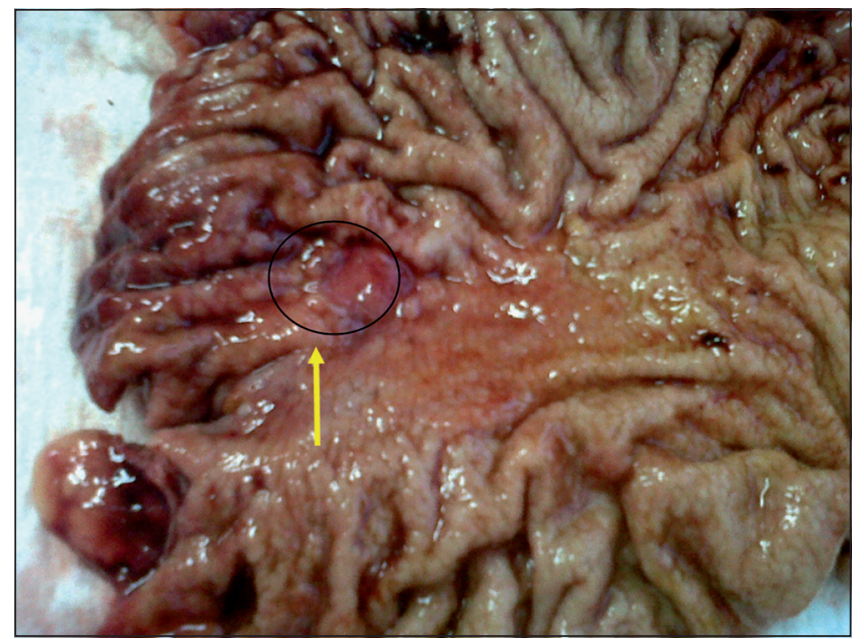

Figura 5. Pieza operatoria, evidencia lesión neoplásica ulcerada en cuerpo gástrico a nivel de la cara anterior, distante $15 \mathrm{~mm}$ de la sección gástrica y 3 $\mathrm{cm}$ del píloro. El tumor medía 3,5 cm en el eje longitudinal y $7 \mathrm{~cm}$ en el transversal, comprometiendo la curvatura menor y extendiéndose $2,5 \mathrm{~cm}$ a la cara posterior. Al corte, estaba constituido por tejido rosado pálido lobulado, liso y brillante, que reemplazaba las túnicas submucosa, muscular propia y subserosa, abombando levemente la serosa.

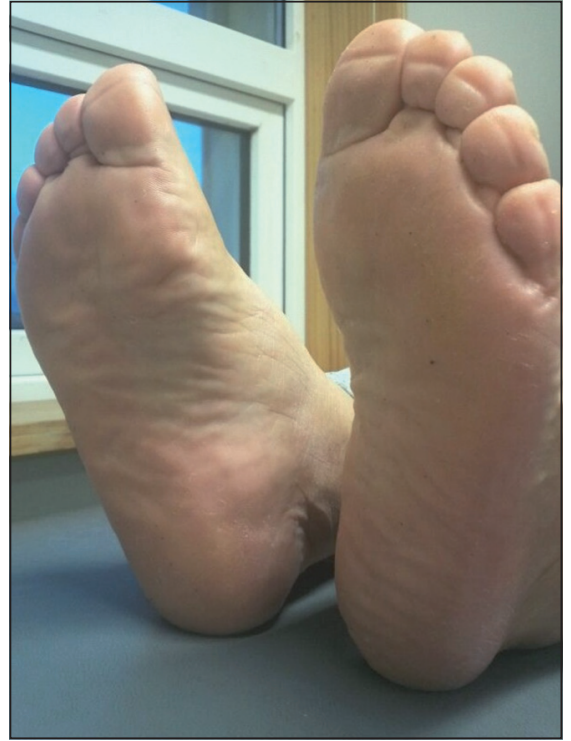

Figura 6. Pies sin lesiones patológicas hiperqueratósicas en relación a lesiones tipo Leser-Trélat.
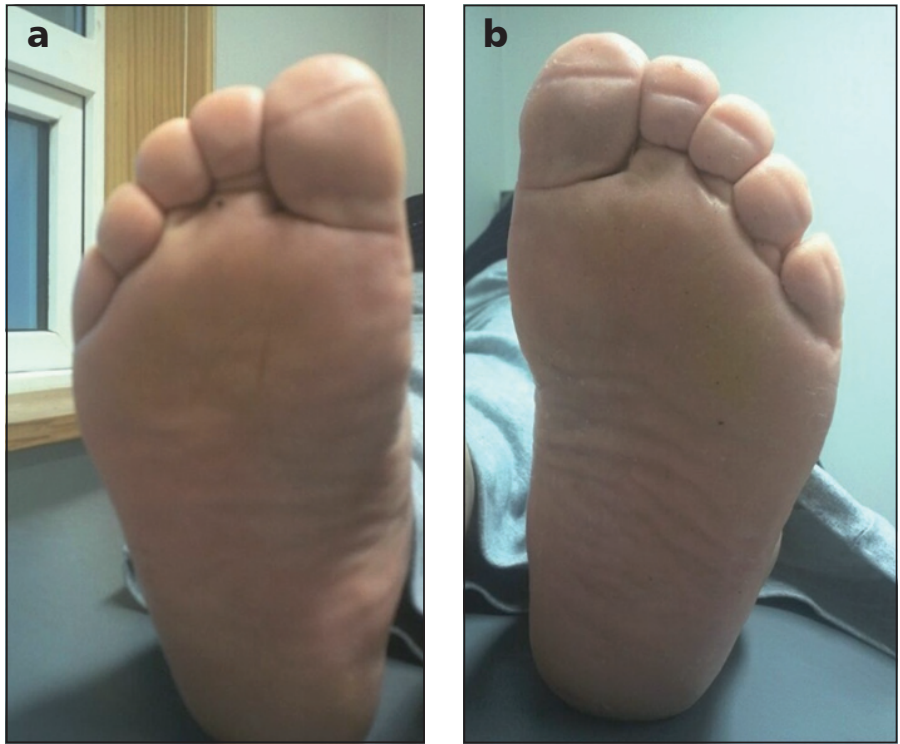

Figura 7. Planta de pie derecho (a) e izquierdo (b) sin lesiones patológicas hiperqueratósicas en relación a lesiones tipo Leser-Trélat.

\section{Discusión}

Los síndromes paraneoplásicos cutáneos corresponden al conjunto de manifestaciones de piel que se presentan antes, durante o después del inicio de una patología tumoral con la que guardan una relación de dependencia no metastásica. Ocupan el segundo lugar de frecuencia entre las manifestaciones paraneoplásicas siendo superadas sólo por las de origen endocrinológico ${ }^{3-5}$.
Los criterios de Curth (1976) mantienen hoy en día plena vigencia y se utilizan para reconocer la asociación entre dermatosis y neoplasia, estos son: comienzo simultáneo, curso paralelo de las dos afecciones, asociación frecuente, especificidad tumoral y rareza de las dermatosis.

Dentro de los síndromes paraneoplásicos encontramos el signo-síndrome de Leser-Trélat, el cual se asocia con mayor frecuencia a cáncer de tipo histológico adenocarcinoma, de los cuales, 
$32 \%$ son de origen gastrointestinal y de ellos uno de los más frecuente es el cáncer gástrico ${ }^{6,7}$.

El signo-síndrome de Leser-Trélat está determinado por la existencia de lesiones tumorales múltiples de tipo seborreicas (queratosis seborreicas), las cuales son benignas y están presentes en un alto porcentaje de la población normal, pero de la que difiere por la aparición de gran cantidad, en forma "explosiva" muy diseminada y pruriginosa (prurito estaría presente en $26 \%$ a $51 \%$ de los casos). Atributos de los que carece la primera forma mencionada. Su patrón de distribución se ha descrito clásicamente como en "árbol de Navidad"7,8.

Estas lesiones representan una proliferación benigna de queratinocitos inmaduros en relación a la explosión múltiple de mediadores inflamatorios en el proceso proliferativo del cáncer gástrico, las alteraciones en los niveles séricos de factores de crecimiento humano, incluyendo el TGF-alfa, TGF-beta, y factor de crecimiento epidermoide (EGF) han sido implicados en la patogénesis pero no probado. Las funciones de factor de crecimiento derivado de plaquetas (PDGF) y el virus del papiloma humano se están explorando9.

Las edades más frecuentemente observadas suelen corresponder a pacientes añosos (promedio de presentación es de 61 años), no existiendo predominancia de raza ni sexo y las lesiones se suelen ubicar en cabeza y tórax y con menor frecuencia en las extremidades a la forma de lesiones pápulo escamosas, de tamaño variable, color café a negro, de superficie verrucoide, untuosa al tacto y de bordes definidos ${ }^{10}$.

Ante la eventualidad de aparición del signo de LT se desprende la necesidad de descartar la presencia de una neoplasia oculta. Dentro de los exámenes propuestos para iniciar este estudio se recomienda realizar bioquímica sanguínea completa, test de Weber, antígeno carcinombrionario, orina completo, antígeno prostático, radiografía de tórax y TAC de abdomen y pelvis. Además, dada su mayor asociación con neoplasias gastrointestinales se sugiere considerar el estudio endoscópico completo del tracto gastrointestinal ${ }^{11}$.

Con respecto al tratamiento, se hace evidente que la respuesta está en la terapéutica de la causa subyacente. En general, el curso del signo es paralelo al de la neoplasia. Por otra parte, se sabe que la persistencia de las lesiones cutáneas traduce fracaso del tratamiento o reactivación de la neoplasia. En general, el pronóstico no es bueno, ya que la mayor parte de las neoplasias asociadas a este signo se comportan de manera agresiva ${ }^{10-12}$.

\section{Referencias}

1. Heise K, Bertrán E, Andia M, Ferreccio C. Incidence and survival of stomach cancer in a high-risk population of Chile. World J Gastroenterol 2009; 15: 1854-62.

2. Crew KD, Neugut AI. Epidemiology of gastric cancer. World J Gastroenterol 2006; 12: 354-62.

3. Guías clínicas GES 2006 para Cáncer Gástrico, Ministerio de Salud-Chile.

4. Pentenero M, Carrozzo M, Pagano M, Gandolfo S. Oral acanthosis nigricans, tripe palms and sign of leser-trélat in a patient with gastric adenocarcinoma. Int J Dermatol. 2004; 43: 530-2.

5. Ishikawa $S$, Togashi A, Inoue M, Honda S, Nozawa F, Toyama E. Indications for EMR/ESD in cases of early gastric cancer: relationship between histological type, depth of wall invasion, and lymph node metastasis. Gastric Cancer 2007; 10: 3.

6. Chung VQ, Moschela SL, Zembowics A, Liu V. Clinical and pathologic findings of paraneoplastic dermatoses. J Am Acad Dermatol. 2006; 54: 745-62.

7. Gadwin-Sánchez F. Signos cutáneos de malignidad interna. Dermatol Perú 2005; 15: 181-5.

8. Boyce S, Harper J. Paraneoplastic dermatoses. Dermatol Clin 2002; 20: 523-32.

9. Viet Q Nguyen et all. Paraneoplastic Diseases. Journal of Ackerman Academy of Dermatopathology, New York, 2011; 26 (1): 17-29.

10. Ghul G, Arruabarrena C, Escalante L, Serrano P. Síndromes paraneoplásicos cutáneos. SEMERGEN 2004; 30: 506-13.

11. Subirat Esquivel L, Guillén Isern D. Algunas consideraciones actuales sobre el Cáncer Gástrico. AMC 2011; 15 (2): 400-11.

12. Ortega-Loayza AG, Ramos W, Gutiérrez Ericson L, Chávez de PP, Bobbio L, Galarza C. Cutaneous manifestations of internal malignancies in a tertiary health care hospital of a developing country. An Bras Dermatol 2010; 85 (5): 736-42. 